\title{
Adaptive Relay Configuration Based on the Novel Hyperchaotic Three-Components Oscillator Operating at High Frequency: Global Synchronization
}

\author{
B. A. Mezatio ${ }^{1,2}$, M. Tingue Motchongom ${ }^{3}$, R. Kengne ${ }^{1,2}$, T. Fozin Fonzin ${ }^{2}$, A. Tchagna Kouanou ${ }^{2}$, \\ R. Tchitnga ${ }^{1,2}$, A. Fomethe ${ }^{4}$ \\ ${ }^{1}$ Department of Physics, Faculty of Science, University of Dschang, Dschang, Cameroon \\ ${ }^{2}$ Laboratory of Electronics and Signal Processing, Department of Physics, Faculty of Science, University of Dschang, Dschang, Cameroon \\ ${ }^{3}$ Higher Technical Teachers Training College, University of Bamenda, Bambili, Cameroon \\ ${ }^{4}$ Unite de Recherche de Mecanique et de Modelisation des Systemes Physique (UR2MSP), Department of Physics, Faculty of Science, \\ University of Dschang, Dschang, Cameroon
}

\section{Email address:}

bricemezat@gmail.com (B. A. Mezatio)

\section{To cite this article:}

B. A. Mezatio, M. Tingue Motchongom, R. Kengne, T. Fozin Fonzin, A. Tchagna Kouanou, R. Tchitnga, A. Fomethe. Adaptive Relay Configuration Based on the Novel Hyperchaotic Three-Components Oscillator Operating at High Frequency: Global Synchronization. Science Journal of Circuits, Systems and Signal Processing. Vol. 9, No. 1, 2019, pp. 1-15. doi: 10.11648/j.cssp.20200901.11

Received: October 8, 2019; Accepted: November 6, 2019; Published: April 13, 2020

\begin{abstract}
This article is investigating from one of best control technique known as periodically intermittent discrete observation control (PIDOC), the problem of global synchronization based on a relay configuration of three novel hyperchaotic oscillators of three-components (NHO) operating at high frequency. Contrary to traditional periodically intermittent control based on continuous-time state observations, PIDOC used here, chooses discrete-time state observations in work time during a control period. Our analysis has been limited to a range of parameters for which the NHO-type oscillator exhibits bursting oscillations. The global conditions of stability for non-adaptive and adaptive cases have been proven analytically. To the best of our knowledge and in the literature of the relay coupling system, no work has been carried out concerning the study of the stability of adaptive synchronization case. The Synchronization of the system is analysed in terms of its control gain by using time series. The numerical results show that there is global synchronization between the three relay coupled NHO-type oscillators for both non-adaptive and adaptive synchronizations. Moreover, PSpice based simulations of the analog electronic circuit for the non-adaptive case are in good accordance with both theoretical and numerical results.
\end{abstract}

Keywords: Bursting Oscillations, High Frequency, Periodically Intermittent Discrete Observation Control, Relay Coupling System, Global Synchronization

\section{Introduction}

The idea of investigating the collective behavior of coupled dynamical systems has provoked a great deal of research efforts in the past two decades $[1,2]$. A primary interest focussed on some phenomena as synchronization, phaselocking, phase-shift, and phase-flip transition, and amplitude death are specially presented in coupled dynamical systems $[2,3,4]$. Of all the above, the synchronization emerges to be the most common phenomenon exhibited in a diversity of coupled dynamical systems. The history of this very interesting phenomenon started in the seventeenth century when the famous researcher Christian Huygens observed perfect similarity between the oscillating motions of two clocks hanging from a same support [5]. Later, Eccles and Vincent [6] analysed synchronization phenomena in the context of electrical and radio engineering development. After the famous works of Pecora and Carroll [7, 8] in which they were able to synchronize two identical chaotic systems with different initial conditions; chaos synchronization owing to its potential application in various fields of physics and engineering sciences, such as information processing, biological systems, artificial neurons networks, chemical 
reactors, data encryption, communications secure, etc [9, 10] has been the subject of remarkable progress. Thus, in the literature different main types of synchronization are usually distinguished, namely complete or identical synchronization (CS), in-phase synchronization (PS), out-ofphase synchronization, lag synchronization (LS), generalized synchronization (GS), intermittent lag synchronization (ILS), and mixed synchronization and so on $[8,11,12,13,14]$. In coupled systems, the synchronization can be achieved either by coupling or external action while considering the nature of coupling, namely: diffusion, time delay, nonlinear, conjugate, as well as strength or intensity of interaction.

However, different techniques have been presented to highlight the synchronization phenomenon in relay coupled systems. Relay synchronization is a process whereby, indirect coupling permits to obtain a complete synchronization of two dynamical systems through a relay unit whose dynamics may be different from that of the synchronous state [2]. For example, during choir practice, the brain serves as a relay of the different vocal cords of the choir members thereby synchronizing their pitches. Many types of works $[2,15$, $16,17,18,19,20,21,22,23,24,25]$ have sufficiently demonstrated the interest (telecommunications, biological and mechanical systems, ...) devoted to the use of the relay between the systems to be synchronized. In [2], the authors have approached a natural question concerning phenomena such as synchronization and amplitude death across the coupling between similar or different variables. The authors of [19] have brought proof of generalized synchronization in systems that act as mediators between two dynamical units that, in turn, show complete synchronization with each other: these are the relay systems. In [21], the modification consists on the fact that the relay unit is modeled to lead the entire network to a desired dynamics, the authors have studied the dynamics of a modified relay-coupled chaotic systems. Moreover, this study shows that the type of synchronization is linked to the sign of the bound of the time derivative of the Lyapunov function. The idea of stabilizing at finite-time a relay coupled scheme of three fractional-order oscillators in the presence of time delay was addressed in [22]. In [23], the phenomenon of partial death in time delay coupled relay system which was done with conjugate coupling by considering Limit-cycle oscillator and R?ssler oscillator was investigated. Later on, the partial death patterns separated by the oscillatory regime in plan parameter was observed, where the outer oscillators are in the oscillatory state and the middle oscillator in the steady state followed by complete death state.

On the other hand, we also noted in the literature that the design of control scheme is often necessary to drive the synchronization of relay coupled systems. Thus to reach this synchronization, many control methods have been developed namely: the impulsive control [26], the feedback control [27], the periodically intermittent control [28], and so on. Among all these control tactics, a particular interest has been carried out on periodically intermittent control which, although being discontinuous, the control also has the particularity that its control inputs are activated during working time and deactivated during rest time. This justifies the fact that it is more economical than those whose control inputs are permanently activated. It is in this sense that the use of periodically intermittent control to synchronize couples systems has yielded many interesting results. This is the case for example of the authors of [24] who designed an appropriate periodically intermittent controller to deal with the synchronization problem of fractional-order memristive neural networks. Contrary to periodically intermittent control used in some Reference as [28, 29] whose work time in a control period is based on continuous-time state observations, Xu's works and collaborators [25] have introduced a novel control technique more reasonable, practical and low-cost, named as periodically intermittent discrete observation control (PIDOC).

Given that the results of the research works mentioned in all the references cited previously are very interesting, there are still open questions of a fundamental nature. To begin with, Most of the circuits/systems used in the relay couplings proposed so far are chaotic and operate in low frequency ranges. Its disadvantage is that its information processing speed in a communication channel will be low because it has been proven in the literature that the more the signal's oscillation frequency used to secure an information is large, the higher the processing speed of this information [30, 31, 32]. Furthermore, All the studies that have been carried out in literature used non-adaptive controllers and the control laws are known. The non-adaptive controller has an inconvenience that when the external milieu varies, it is not robust enough to secure the synchronization of the systems put in play because its gain is manual and consequently unable to adapt to environmental modifications. To continue, the majority of the relay systems don't take into consideration the effect of the transmission channel. Given that, there is no existence of a perfect channel, not taking into consideration is only utopia. To ride on, still in the literature of relay systems, we also noticed that it is poor as of what concerns the study of stability. It is important to note that to avoid losing information, the synchronization phenomenon should be anticipated through calculation. Indeed, final-time synchronization permits to accurately anticipate the time of synchronization in a system. This time synchronization is important for an engineer because it permits a better determination of parameters which can entail the synchronization of coupled systems [33]. In this context, there is a keen work in the study of finite-time chaos synchronization [34, 35] and gradually of fractionalorder systems $[33,36,37]$. To the best of our knowledge, except some works like those defined in [21], [22] and more recently in [25] to mention only those who studied stability in the non-adaptive case, no further work has been done in respect to adaptive synchronization case. since the adaptive method has the advantage of making the synchronization process more robust to disturbances and uncertainties related to the parameters and the initial conditions of the system. Recall that the problems faced in the literature of relay coupled systems and underlined in this paragraph have been summarised in Table 1. 
Motivated by the above discussions and via PIDOC, we study the problem of synchronization in a relay configuration based on the coupling of three new three-component hyperchaotic oscillators (NHO) whose synchronization criteria are obtained with the help of Lyapunov method. We have also succeeded in constructing a Lyapunov function that could reflect the topological structure of our relay configuration. Moreover, sufficient conditions to ensure global synchronization of the outer oscillators are presented for both the non-adaptive synchronization and the adaptive synchronization. This paper is organized as follows: A brief reminder of the NHO-type oscillator followed by the model description of relay systems as well as some preliminary are given in Section 2. Section 3 is devoted to the main results of the stability study corresponding to the non-adaptive synchronization and adaptive synchronization respectively in a relay coupling of three NHO-type oscillators. Numerical simulations are carried out in Section 4. PSpice simulations are performed in Section 5 to illustrate the effectiveness of the relay coupling of three NHO-type oscillators. We complete the paper in Section 6 with an appropriate conclusion and some further works.

Table 1. Classification of some systems in terms of problems faced in this study.

\begin{tabular}{|c|c|c|c|c|c|}
\hline $\begin{array}{l}\text { Referemce } \\
\text { of papers }\end{array}$ & Hyperchaos & $\begin{array}{l}\text { High } \\
\text { frequency }\end{array}$ & $\begin{array}{l}\text { Periodically intermittent discrete } \\
\text { observation control (PIDOC) }\end{array}$ & $\begin{array}{l}\text { Stability study for the case of } \\
\text { nonadaptive synchronization }\end{array}$ & $\begin{array}{l}\text { Stability study for the case of } \\
\text { adaptive synchronization }\end{array}$ \\
\hline [2] & No & No & No & No & No \\
\hline [17] & No & No & No & No & No \\
\hline [19] & No & No & No & No & No \\
\hline [21] & No & No & No & Yes & No \\
\hline [22] & No & No & No & Yes & No \\
\hline [23] & No & No & No & No & No \\
\hline [28] & No & No & No & Yes & No \\
\hline [29] & No & No & No & Yes & No \\
\hline This work & Yes & Yes & Yes & Yes & Yes \\
\hline
\end{tabular}

\section{Novel Hyperchaotic Three-component Oscillator (NHO) Description and Preliminaries}

\subsection{NHO Description}

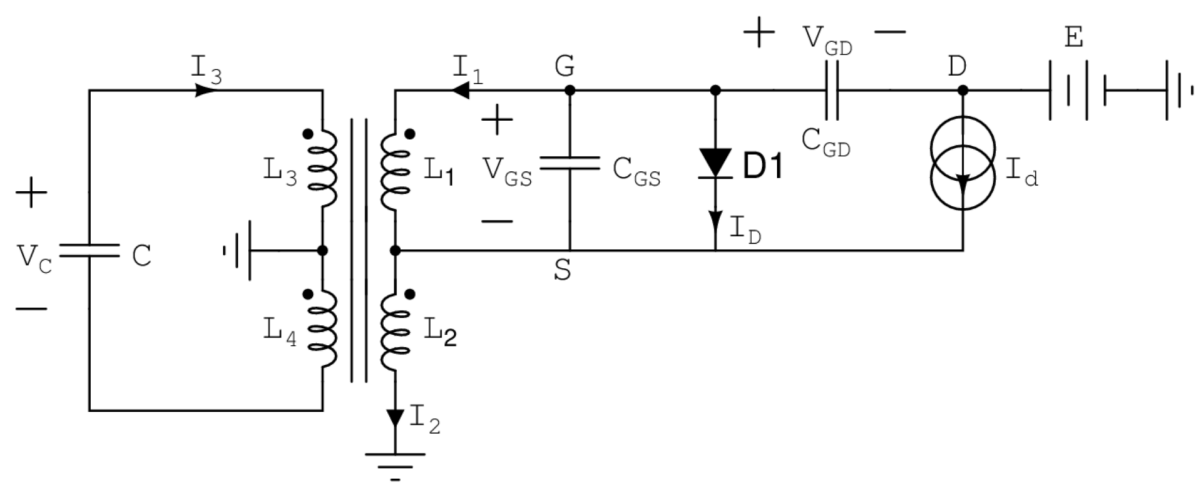

Figure 1. Schematic representation of NHO governed by the six-dimensional flow in Eq. (1).

As mentioned, Figure 1 presented in this work contains only three elements, namely a junction field effect transistor (JFET), a transformer and a capacitor and was analysed experimentally by Tchitnga et al. [32]. In order to facilitate the modeling of the $\mathrm{NHO}$, these authors have taken into consideration certain modifications, namely, they neglected the internal resistance of the tapped coil and used a high-frequency small-signal equivalent circuit model of a JFET, as described in their paper. Then, the equations governing the circuit are given in Eq. (1)

$$
\left\{\begin{array}{l}
\frac{d V_{G S}}{d t}=\frac{1}{c_{g s}}\left(i_{2}-I_{d}-i_{1}-i_{D}\right) \\
\frac{d V_{G D}}{d t}=\frac{1}{c_{g d}}\left(-i_{2}+I_{d}\right) \\
\frac{d V_{C}}{d t}=-\frac{1}{c} i_{3} \\
V_{G S}=L_{1} \frac{d i_{1}}{d t}+M_{12} \frac{d i_{2}}{d t}+\left(M_{13}-M_{14}\right) \frac{d i_{3}}{d t} \\
E+V_{G D}-V_{G S}=M_{21} \frac{d i_{1}}{d t}+L_{2} \frac{d i_{2}}{d t}+\left(M_{23}-M_{24}\right) \frac{d i_{3}}{d t} \\
V_{C}=\left(M_{31}-M_{41}\right) \frac{d i_{1}}{d t}+\left(M_{32}-M_{42}\right) \frac{d i_{2}}{d t}+\left(L_{3}+L_{4}-M_{34}-M_{43}\right) \frac{d i_{3}}{d t}
\end{array}\right.
$$


where the mutual inductances between $L_{1}$ and $L_{2}, L_{1}$ and $L_{3}, L_{1}$ and $L_{4}, L_{2}$ and $L_{3}, L_{2}$ and $L_{4}, L_{3}$ and $L_{4}$ are defined as $M_{12}=M_{21}=k_{1} \sqrt{L_{1} L_{2}}, M_{13}=M_{31}=k_{2} \sqrt{L_{1} L_{3}}, M_{14}=M_{41}=k_{3} \sqrt{L_{1} L_{4}}, M_{23}=M_{32}=k_{4} \sqrt{L_{2} L_{3}}, M_{24}=M_{42}=$ $k_{5} \sqrt{L_{2} L_{4}}$ and $M_{34}=M_{43}=k_{6} \sqrt{L_{3} L_{4}}$ respectively, where $k_{i}(i=1, \ldots, 6)$ are the magnetic coupling factors.

Also recall that the currents are:

$$
\begin{aligned}
& I_{d}=\left\{\begin{array}{lc}
0, & \text { if } V_{G S} \leq V_{G S o f f}, \\
g_{m 0}\left(V_{G S}-V_{G S o f f}\right)^{2}, & \text { if } V_{G D} \leq V_{G S o f f}, \\
g_{m 0}\left(V_{G S}-V_{G D}\right)\left(V_{G S}+V_{G D}-2 V_{G S o f f}\right), & \text { if } V_{G D}, \geq V_{G S o f f}
\end{array} .\right. \\
& i_{D}=I_{S}\left[\exp \left(\frac{V_{G S}}{V_{T}}\right)-1\right]
\end{aligned}
$$

As a reminder, Eq. (1) describing the circuit system of Figure 1 whose its normalized form is presented in [32] and defined as follow:

$$
\left\{\begin{array}{l}
\dot{x}_{1}=a_{1}\left(x_{5}-x_{4}\right)-a_{2}\left[\exp \left(x_{1}\right)-1\right]-a_{3} g\left(x_{1}, x_{2}\right) \\
\dot{x}_{2}=-\alpha\left(a_{1} x_{5}-a_{3} g\left(x_{1}, x_{2}\right)\right) \\
\dot{x}_{3}=-a_{4} x_{6} \\
\dot{x}_{4}=b_{1} x_{1}+b_{2}\left(e+x_{2}\right)+b_{3} x_{3} \\
\dot{x}_{5}=b_{4} x_{1}+b_{5}\left(e+x_{2}\right)+b_{6} x_{3} \\
\dot{x}_{6}=b_{7} x_{1}+b_{8}\left(e+x_{2}\right)+b_{9} x_{3}
\end{array}\right.
$$

where $x_{1}, x_{2}, x_{3}, x_{4}, x_{5}$ and $x_{6}$ are the dimensionless states variables and $\tau=\omega_{0} t\left(\omega_{0}\right.$ is the resonant radian frequency of the system). The constant parameters $a_{i}(i=1, \ldots, 4), b_{j}(j=1, \ldots, 9), \alpha$ and $e$ are given by the following relations:

$$
\begin{gathered}
a_{1}=\frac{I_{0}}{V_{T} C_{G S} \omega_{0}}, a_{2}=\frac{I_{S}}{V_{T} C_{G S} \omega_{0}}, a_{3}=\frac{g_{m 0} V_{T}}{C_{G S} \omega_{0}}, a_{4}=\frac{I_{0}}{V_{T} C \omega_{0}}, b_{1}=\frac{V_{T}\left(a_{11}-a_{12}\right)}{I_{0} \theta \omega_{0}}, \\
b_{2}=\frac{V_{T} a_{12}}{I_{0} \theta \omega_{0}}, b_{3}=\frac{V_{T} a_{13}}{I_{0} \theta \omega_{0}}, b_{4}=\frac{V_{T}\left(a_{21}-a_{22}\right)}{I_{0} \theta \omega_{0}}, b_{5}=\frac{V_{T} a_{22}}{I_{0} \theta \omega_{0}}, \\
b_{6}=\frac{V_{T} a_{23}}{I_{0} \theta \omega_{0}}, b_{7}=\frac{V_{T}\left(a_{31}-a_{32}\right)}{I_{0} \theta \omega_{0}}, b_{8}=\frac{V_{T} a_{32}}{I_{0} \theta \omega_{0}}, b_{9}=\frac{V_{T} a_{33}}{I_{0} \theta \omega_{0}}, \alpha=\frac{C_{G S}}{C_{G D}}, \text { and } e=\frac{E}{V_{T}} .
\end{gathered}
$$

with

$$
\begin{gathered}
\theta=L_{1} L_{2}\left[\begin{array}{c}
2\left(k_{1} k_{2} k_{5}+k_{1} k_{3} k_{4}-k_{1} k_{1} k_{6}-k_{2} k_{3}-k_{4} k_{5}+k_{6}\right) \\
\sqrt{L_{3} L_{4}}+\left(-2 k_{1} k_{2} k_{4}+k_{1} k_{1}+k_{2} k_{2}+k_{4} k_{4}-1\right) L_{3}+\left(-2 k_{1} k_{3} k_{5}+k_{1} k_{1}+k_{3} k_{3}+k_{5} k_{5}-1\right) L_{4}
\end{array}\right] \\
a_{11}=\left[\left(k_{4} k_{4}-1\right) L_{3}+\left(k_{5} k_{5}-1\right) L_{4}+2\left(k_{6}-k_{4} k_{5}\right) \sqrt{L_{3} L_{4}}\right] L_{2} \\
a_{22}=\left[\left(k_{2} k_{2}-1\right) L_{3}+\left(k_{3} k_{3}-1\right) L_{4}+2\left(k_{6}-k_{2} k_{3}\right) \sqrt{L_{3} L_{4}}\right] L_{1} \\
a_{33}=\left(k_{1} k_{1}-1\right) L_{1} L_{2} \\
a_{12}=a_{21}=\left[\left(-k_{2} k_{4}+k_{1}\right) L_{3}+\left(-k_{3} k_{5}+k_{1}\right) L_{4}+\left(-2 k_{1} k_{6}+k_{2} k_{5}+k_{3} k_{4}\right) \sqrt{L_{3} L_{4}}\right] \sqrt{L_{1} L_{2}} \\
a_{13}=a_{31}=\left[\left(-k_{1} k_{4}+k_{2}\right) \sqrt{L_{1} L_{3}}+\left(k_{1} k_{5}-k_{3}\right) \sqrt{L_{1} L_{4}}\right] L_{2} \\
a_{23}=a_{32}=\left[\left(k_{3} k_{1}-k_{5}\right) \sqrt{L_{2} L_{4}}+\left(k_{4}-k_{1} k_{2}\right) \sqrt{L_{2} L_{3}}\right] L_{1}
\end{gathered}
$$

Let's also note that all the coefficients $a_{i j}(i=1, \ldots, 3$, $j=1, \ldots, 3)$ as for them, derive from the parameters of the midpoint transformer namely, the mutual inductances $L_{p}$ $(p=1, \ldots, 4)$ as well as the magnetic coupling factors $k_{q}$ $(q=1, \ldots, 6)$ [32]. Of most interesting is the observation that the system (2) not only can display hyperchaotic attractors when $k_{1}=0.90, k_{2}=0.93, k_{3}=0.82, k_{4}=0.93$, $k_{5}=0.84, k_{6}=0.93$ and $e=41.5$, but also can exhibits a rich dynamic behaviors intrinsic to neurons, characterized of alternating sequence of active states and passive states, and known as bursting oscillations when $e=25$ (see Figure 2).

\subsection{Relay Circuit and Equation}

With the aim of determining whether a relay configuration based on the coupling of three NHO-type oscillators is possible, let consider diagram is given in Figure 3. The arrows 
between the oscillators indicate that the coupling is assumed to be bidirectional and instantaneous. Each of the oscillators of Figure 3 is a NHO. Here, the relay configuration is chosen so that the three oscillators are all taken to be identical except for the initial conditions. Here, the issue is to establish a connection between the node $X$ and the node $Z$ through the node $Y$ considered in this work as the relay. On the basis of the mathematical model of NHO as defined above, we can write the equations of the nodes $X, Y$ and $Z$ respectively in the following way: the outer 1 oscillator

$$
\left\{\begin{array}{l}
\dot{X}=F(X, t)+u_{1}\left(\delta_{\tau}\right) \\
\text { where } \quad \mathrm{X}=\left(x_{1}, x_{2}, x_{3}, x_{4}, x_{5}, x_{6}\right)^{T}
\end{array}\right.
$$

the relay oscillator

$$
\left\{\begin{array}{l}
\dot{Y}=F(Y, t)+u_{2}\left(\delta_{\tau}\right) \\
\text { where } \mathrm{Y}=\left(y_{1}, y_{2}, y_{3}, y_{4}, y_{5}, y_{6}\right)^{T}
\end{array}\right.
$$

and the outer 2 oscillator

$$
\left\{\begin{array}{l}
\dot{Z}=F(Z, t)+u_{3}\left(\delta_{\tau}\right) \\
\text { where } \quad \mathrm{Z}=\left(z_{1}, z_{2}, z_{3}, z_{4}, z_{5}, z_{6}\right)^{T}
\end{array}\right.
$$

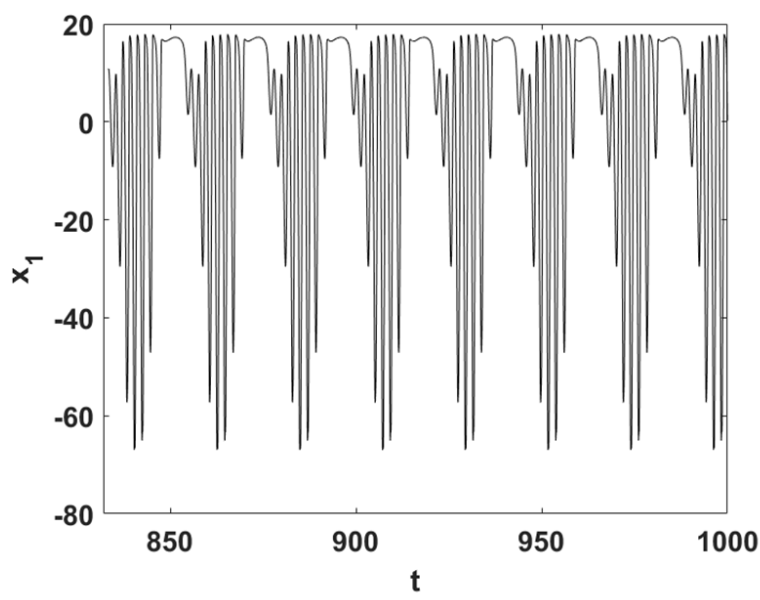

(a) where $u_{1}$ represents the controller between node $X$ and node $Y, u_{3}$ represents the controller between node $Z$ and node $Y$ and $u_{2}$ the inverse signal of the sum of $u_{1}$ et $u_{2}$ which comes from comtributions of the node $X$ and $Y$. Furthermore, $X(t)=\left(x_{1}(t), x_{2}(t), x_{3}(t), x_{4}(t), x_{5}(t), x_{6}(t)\right)^{T} \in$ $\mathbb{R}^{n}$ corresponds to the vector of the node $X$, $Y(t)=\left(y_{1}(t), y_{2}(t), y_{3}(t), y_{4}(t), y_{5}(t), y_{6}(t)\right)^{T} \in \mathbb{R}^{n}$ corresponds to the vector of the node $Y$ and $Z(t)=$ $\left(z_{1}(t), z_{2}(t), z_{3}(t), z_{4}(t), z_{5}(t), z_{6}(t)\right)^{T} \in \mathbb{R}^{n}$ corresponds to the vector of the node $Z$. Here, $F(\cdot): \mathbb{R}^{n} \rightarrow \mathbb{R}^{n}$ is a continuous vector-valued function

To ensure global synchronization, we choose the dynamic synchronization error described by:

$$
e(t)=X(t)+Z(t)-2 Y(t)
$$

So the dynamics of this error can be expressed as follows:

$$
\dot{e}(t)=F(X)+F(Z)-2 F(Y)+u_{i}(t)
$$

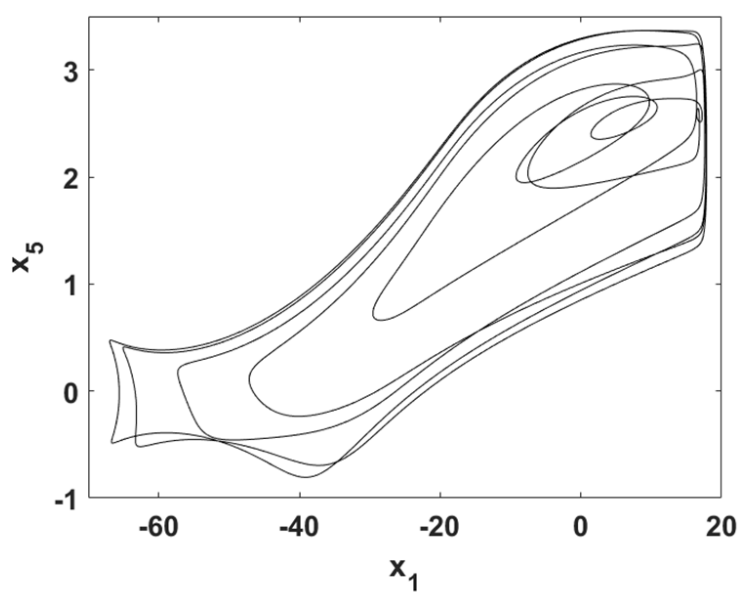

(b)

Figure 2. Periodic bursting: (a) time serie of the output of $x_{1}$ and $(b) x_{5}$ phase space projection in the plane $\left(x_{1}-x_{5}\right)$ for $k_{1}=0.90, k_{2}=0.93, k_{3}=0.82, k_{4}=0.93, k_{5}=0.84, k_{6}=0.93$ and $e=25$. Initial conditions $X(0)=$ $(0.001,0.008,0.004,0.004,0.005,0.001)^{T}$.

\subsection{Preliminaries}

Some definitions are presented in order to prepare for main results.

Definition 1. If in the case of non-adaptive synchronization $\lim _{t \rightarrow \infty}|e(t)|=0$, a global synchronization is achieved between

$$
d_{i}(t)=\left\{\begin{array}{l}
d_{i}, \quad t \in[m T,(m+\theta) T] \\
0, \quad t \in[(m+\theta) T,(m+1) T], m \in \mathbb{N}
\end{array}\right.
$$

With $T>0$ represents the control period, $d_{i}$ is a positive constant called the control gain, $\theta T$ is called the control width. Furthermore, $\delta_{\tau}=[t / \tau] \tau-\tau=l \tau-\tau$, where positive constant $\tau$ is the duration between two consecutive observations and outer 1, outer 2 oscillators and the relay oscillator.

In order to make system (7) reach global synchronization, a PIDOC [25] $u_{i}(t)$ who in the non-adaptive case is designed as:

$$
u_{i}(t)=-d_{i}(t) e\left(\delta_{\tau}\right)
$$

in which

$l=[t / \tau]$ is the integer part of $t / \tau$. Meanwhile, $\theta T / \tau$ is a positive integer.

Definition 2. If in the case of adaptive synchronization 


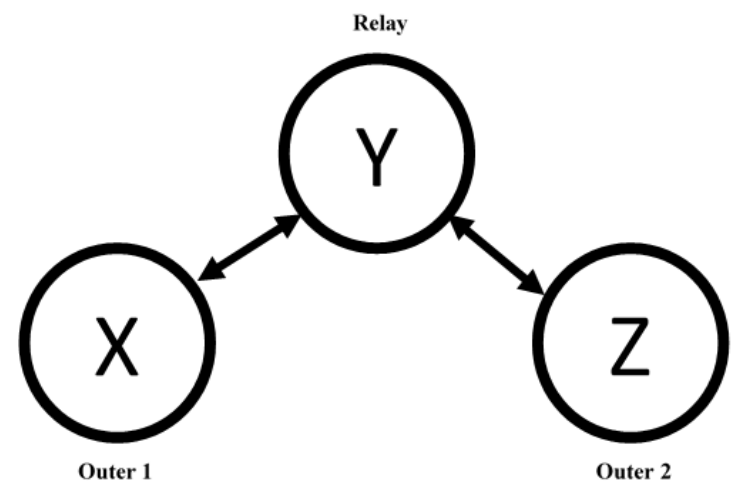

Figure 3. Relay configuration scheme in the present model.

$\lim _{t \rightarrow t_{r}}|e(t)|=0$, between the outer 1 , outer 2 oscillators and relay unit, a global synchronization at finite time $t_{r}$ is achieved.

For the system (7) to reach global synchronization in the

$$
\dot{d}_{i}(t)=\left\{\begin{array}{l}
(e(t))^{2}, \quad t \in[m T,(m+\theta) T] \\
0, \quad t \in[(m+\theta) T,(m+1) T], m \in \mathbb{N}
\end{array}\right.
$$

in which $T>0$ represents the control period, $d_{i}$ is the control gain, $\theta T$ is called the control width. Furthermore, $\delta_{\tau}=$ $[t / \tau] \tau-\tau$, where $\tau$ is the duration between two consecutive observations and $[t / \tau]$ is the integer part of $t / \tau$. Meanwhile, $\theta T / \tau$ is a positive integer.

To deduce main results, some lemmas are given which will be used in main results. adaptive case whose PIDOC is given to the relation (8), choose the PIDOC gain update law as shown below:

Lemma $1[38,39]$. Let $n \in$ and let $q_{1}, q_{2}, \ldots, q_{n}$ be nonnegative real numbers. Then for $z>1$,

$$
\left(\sum_{i=1}^{n} q_{i}\right)^{z} \leq n^{z-1} \sum_{i=1}^{n} q_{i}^{z}
$$

Lemma 2[38, 22]. (Gronwall inequality) . If

$$
y(t) \leq v(t)+\int_{t_{0}}^{t} h(s) y(s) d s \quad t \in\left[t_{0}, T\right]
$$

$\left[t_{0}, T\right], \quad T \leq \infty$ and $h(s) \geq 0$, then $y(t)$ satisfies

$$
y(t) \leq v(t)+\int_{t_{0}}^{t} h(s) y(s) \exp \left[\int_{s}^{t} h(u) d u\right] d s \quad t \in\left[t_{0}, T\right]
$$

If, in addition, $v(t)$ is nondecreasing, then

$$
y(t) \leq v(t) \exp \int_{t_{0}}^{t} h(s) d s \quad t \in\left[t_{0}, T\right]
$$

Lemma 3 [22]. If $F$ is Leibchitzian function $(F(0)=0)$, then it exist a constant $\eta$ such as:

$$
|F(X)+F(Z)-2 F(Y)| \leq \eta|e|
$$

\section{Main Result}

\subsection{Non-adaptive Synchronization Based on the Relay Coupling of Three NHO-type Oscillators}

Now let's interest us at the stability of the error system (7). Therefore, (7) combined with (8) leads to:

$$
\dot{e}=F(X)+F(Z)-2 F(Y)-d_{i}(t) e\left(\delta_{\tau}\right)
$$

Let $d_{i}=\bar{d}$ the control gain. This system (15) is stable if and only if the control gain $\bar{d}$ satisfies Theorem 1

Theorem 1. If $(\bar{d}-2 \eta)(1-p(\tau))-d p(\tau) \geq 0$, i.e,

$$
8 \tau^{2} \bar{d}^{3} \exp \left(4 \tau^{2} \eta^{2}\right)-8 \eta \tau^{2} \bar{d}^{2} \exp \left(4 \tau^{2} \eta^{2}\right)+16 \tau^{2} \eta^{2} \bar{d} \exp \left(4 \tau^{2} \eta^{2}\right)-16 \tau^{2} \eta^{3} \exp \left(4 \tau^{2} \eta^{2}\right)-\bar{d} \leq 2 \eta
$$


In this hypothesis, we say that there is global synchronization between the three oscillators namely: $X, Y$ and $Z$ Proof. To prove this theorem, consider the following Lyapunov function:

$$
V(t)=e^{T}(t) e(t)
$$

its time derivative leads us to the relation below:

$$
\begin{aligned}
\dot{V}_{i}=2 e^{T} \dot{e} & \\
& =2 e^{T}(F(X)+F(Z)-2 F(Y))+2 e^{T} u_{i}(t)
\end{aligned}
$$

By applying the Lemma 3 to Eq.(18), we have

$$
\dot{V} \leq 2 \eta|e(t)|^{2}-2 d_{i}(t) e(t) e\left(\delta_{\tau}\right)
$$

Given that $d_{i}=\bar{d}$, we obtain:

$$
\begin{gathered}
\dot{V} \leq 2 \eta|e(t)|^{2}+\bar{d}\left(e(t)-e\left(\delta_{\tau}\right)\right)^{2}-\bar{d} e^{2}(t) \\
\dot{V} \leq(2 \eta-\bar{d})|e(t)|^{2}+\bar{d}\left[e(t)-e\left(\delta_{\tau}\right)\right]^{2}
\end{gathered}
$$

Let us evaluate, $\left|e(t)-e\left(\delta_{\tau}\right)\right|^{2}$

$$
e(t)-e\left(\delta_{\tau}\right) \leq \int_{\delta_{\tau}}^{t} d e \leq \int_{\delta_{\tau}}^{t} \dot{e}(s) d s
$$

Seen that $\tau=t-\delta_{\tau}$, it yields that

$$
\left|e(t)-e\left(\delta_{\tau}\right)\right|^{2} \leq \tau \int_{\delta_{\tau}}^{t}\left|f\left(x_{i}\right)+f\left(z_{i}\right)-2 f\left(y_{i}\right)-d(s) e\left(\delta_{\tau}\right)\right|^{2} d s
$$

Using Lemma 1, the inequality (23) can take the following form:

$$
\left|e(t)-e\left(\delta_{\tau}\right)\right|^{2} \leq 2 \tau \int_{\delta_{\tau}}^{t}\left|f\left(x_{i}\right)+f\left(z_{i}\right)-2 f\left(y_{i}\right)\right|^{2} d s+2 \tau \int_{\delta_{\tau}}^{t}|d(s)|^{2}\left|e\left(\delta_{\tau}\right)\right|^{2} d s
$$

According to Lemma 3 the inequality (24), we get

$$
\begin{gathered}
\left|e(t)-e\left(\delta_{\tau}\right)\right|^{2} \leq 2 \tau \eta^{2} \int_{\delta_{\tau}}^{t}|e(s)|^{2} d s+2 \tau^{2} d^{2}\left|e\left(\delta_{\tau}\right)\right|^{2} \\
\left|e(t)-e\left(\delta_{\tau}\right)\right|^{2} \leq 2 \tau \eta^{2} \int_{\delta_{\tau}}^{t}\left|e(s)-e\left(\delta_{\tau}\right)+e\left(\delta_{\tau}\right)\right|^{2} d s+2 \tau^{2} d^{2}\left|e\left(\delta_{\tau}\right)\right|^{2} \\
\left|e(t)-e\left(\delta_{\tau}\right)\right|^{2} \leq \int_{\delta_{\tau}}^{t}\left(4 \tau \eta^{2}\right)\left|e(s)-e\left(\delta_{\tau}\right)\right|^{2} d s+\left(4 \tau^{2} \eta^{2}+2 \tau^{2} d^{2}\right)\left|e\left(\delta_{\tau}\right)\right|^{2}
\end{gathered}
$$

With the help of Lemma 2 on the inequality (27), it follows that:

$$
\begin{gathered}
\left|e(t)-e\left(\delta_{\tau}\right)\right|^{2} \leq\left(4 \tau^{2} \eta^{2}+2 \tau^{2} d^{2}\right)\left|e\left(\delta_{\tau}\right)\right|^{2} \exp \left(4 \tau^{2} \eta^{2}\right) \\
\left|e(t)-e\left(\delta_{\tau}\right)\right|^{2} \leq\left(4 \tau^{2} \eta^{2}+2 \tau^{2} d^{2}\right)\left(\left|e\left(\delta_{\tau}\right)-e(t)+e(t)\right|^{2}\right) \exp \left(4 \tau^{2} \eta^{2}\right) \\
\left|e(t)-e\left(\delta_{\tau}\right)\right|^{2} \leq\left(4 \tau^{2} \eta^{2}+2 \tau^{2} d^{2}\right)\left(2\left|e(t)-e\left(\delta_{\tau}\right)\right|^{2}+2|e(t)|^{2}\right) \exp \left(4 \tau^{2} \eta^{2}\right) \\
\left|e(t)-e\left(\delta_{\tau}\right)\right|^{2} \leq\left\{2\left(4 \tau^{2} \eta^{2}+2 \tau^{2} d^{2}\right)\left|e(t)-e\left(\delta_{\tau}\right)\right|^{2}+2\left(4 \tau^{2} \eta^{2}+2 \tau^{2} d^{2}\right)|e(t)|^{2}\right\} \exp \left(4 \tau^{2} \eta^{2}\right)
\end{gathered}
$$




$$
\left|e(t)-e\left(\delta_{\tau}\right)\right|^{2} \leq p(\tau)\left|e(t)-e\left(\delta_{\tau}\right)\right|^{2}+p(\tau)|e(t)|^{2}
$$

with

$$
p(\tau)=2\left(4 \tau^{2} \eta^{2}+2 \tau^{2} d^{2}\right) \exp \left(4 \tau^{2} \eta^{2}\right)
$$

we can derive that

$$
\begin{gathered}
(1-p(\tau))\left|e(t)-e\left(\delta_{\tau}\right)\right|^{2} \leq p(\tau)|e(t)|^{2} \\
\left|e(t)-e\left(\delta_{\tau}\right)\right|^{2} \leq \frac{p(\tau)}{(1-p(\tau))}|e(t)|^{2}
\end{gathered}
$$

In view of $(1-p(\tau))>0$ and substituting (34) into (21), we obtain:

$$
\begin{gathered}
\dot{V} \leq(2 \eta-\bar{d})|e(t)|^{2}+\frac{\bar{d} p(\tau)}{[1-p(\tau)]}|e(t)|^{2} \\
\dot{V} \leq\left(2 \eta-\bar{d}+\frac{\bar{d} p(\tau)}{[1-p(\tau)]}\right)|e(t)|^{2} \\
\dot{V} \leq-\left(\bar{d}-2 \eta-\frac{\bar{d} p(\tau)}{[1-p(\tau)]}\right)|e(t)|^{2}
\end{gathered}
$$

Set: $\mu=\left(\bar{d}-2 \eta-\frac{\bar{d} p(\tau)}{[1-p(\tau)]}\right)$

$$
\dot{V} \leq-\mu V
$$

The error system (15) is stable if and only if, $\mu \geq 0$, i.e, $\bar{d}-2 \eta-\frac{\bar{d} p(\tau)}{[1-p(\tau)]} \geq 0$

This implies that

$$
\begin{gathered}
(\bar{d}-2 \eta)(1-p(\tau))-\bar{d} p(\tau) \geq 0 \\
\bar{d}-4 \bar{d}\left(4 \tau^{2} \eta^{2}+2 \tau^{2} \bar{d}^{2}\right) \exp \left(4 \tau^{2} \eta^{2}\right)+4\left(4 \tau^{2} \eta^{2}+2 \tau^{2} \bar{d}^{2}\right) \exp \left(4 \tau^{2} \eta^{2}\right) \geq 2 \eta
\end{gathered}
$$

Therefore:

$$
8 \tau^{2} \bar{d}^{3} \exp \left(4 \tau^{2} \eta^{2}\right)-8 \eta \tau^{2} \bar{d}^{2} \exp \left(4 \tau^{2} \eta^{2}\right)+16 \tau^{2} \eta^{2} \bar{d} \exp \left(4 \tau^{2} \eta^{2}\right)-16 \tau^{2} \eta^{3} \exp \left(4 \tau^{2} \eta^{2}\right)-\bar{d} \leq 2 \eta
$$

By assumption: The condition of Theorem 1 hold, so system (15) can reach global synchronization i.e, it can have global synchronization between oscillators $X, Y$ and $Z$ coupled in relay.

Remark: If $\delta_{\tau} \rightarrow t$, one has: $p(\tau) \rightarrow 0$ and $\bar{d}-2 \eta>0$, then

$$
\bar{d}>2 \eta
$$

\subsection{Adaptive Synchronization Based on the Relay}

\section{Coupling of Three NHO-type Oscillators}

Here, let remind that the PODIC gain update Law as well as the dynamics of the error were respectively given by the relations (9) and (15).

Theorem 2. There is global synchronization between the three oscillators if and only if, the following condition is satisfied:

$$
t_{r}=t_{0}+\frac{\ln \left|V\left(t_{0}\right)-\frac{\alpha}{\beta}\right|-\ln \left|V\left(t_{r}\right)-\frac{\alpha}{\beta}\right|}{\beta}
$$

Proof. Choose the following Lyapunov function:

$$
V=e^{T} e+\frac{1}{2}\left(d_{i}-\bar{d}\right)^{2}
$$

Differentiating the Lyapunov function $V$ with respect to time yields

$$
\dot{V}=2 e^{T} \dot{e}+\dot{d}_{i}\left(d_{i}-\bar{d}\right)
$$


By analogy with the non-adaptive case, the relation (45) becomes:

$$
\dot{V} \leq 2 \eta e^{2}(t)+d_{i}(t)\left(e(t)-e\left(\delta_{\tau}\right)\right)^{2}-d_{i}(t) e(t)-d_{i}(t) e\left(\delta_{\tau}\right)+e^{2}(t)\left(d_{i}-\bar{d}\right)
$$

The inequality (46) lead us to the following inequality:

$$
\dot{V} \leq 2 \eta e^{2}(t)+d_{i}(t)\left(e(t)-e\left(\delta_{\tau}\right)\right)^{2}-\bar{d} e^{2}(t)
$$

$d_{i}(t)$ converges to reach $\bar{d}$. So, if $d_{i}(t)$ is an increasing and positive function then $\bar{d}=\max \left(d_{i}(t)\right)$ inequality (47) becomes

$$
\dot{V} \leq(2 \eta-\bar{d}) e^{2}(t)+\bar{d}\left[e(t)-e\left(\delta_{\tau}\right)\right]^{2}
$$

substituting (35) into (48) leads to:

$$
\dot{V} \leq\left[2 \eta-\bar{d}+\frac{p(\tau)}{(1-p(\tau))}\right]|e(t)|^{2}
$$

Then

$$
\dot{V} \leq\left[2 \eta-\bar{d}+\frac{\bar{d} p(\tau)}{(1-p(\tau))}\right]\left(|e(t)|^{2}+\frac{1}{2}\left(d_{i}-\bar{d}\right)^{2}-\frac{1}{2}\left(d_{i}-\bar{d}\right)^{2}\right)
$$

This implies that

$$
\begin{gathered}
\dot{V} \leq\left[2 \eta-\bar{d}+\frac{\bar{d} p(\tau)}{(1-p(\tau))}\right] V-\left[2 \eta-\bar{d}+\frac{\bar{d} p(\tau)}{(1-p(\tau))}\right]\left(\frac{1}{2}\right)\left(d_{i}-\bar{d}\right)^{2} \\
\dot{V} \leq-\left[\bar{d}-2 \eta-\frac{\bar{d} p(\tau)}{(1-p(\tau))}\right] V+\left[2 \eta-\bar{d}+\frac{\bar{d} p(\tau)}{(1-p(\tau))}\right]\left|\frac{d_{i}-\bar{d}}{\sqrt{2}}\right|^{2}
\end{gathered}
$$

In order for it to synchronize, it would be necessary:

$$
\left[2 \eta-\bar{d}+\frac{\bar{d} p(\tau)}{(1-p(\tau))}\right]<0
$$

Let

$$
\alpha=\left[2 \eta-\bar{d}+\frac{\bar{d} p(\tau)}{(1-p(\tau))}\right]\left|\frac{d_{i}-\bar{d}}{\sqrt{2}}\right|^{2}
$$

and

$$
\beta=\bar{d}-2 \eta-\frac{\bar{d} p(\tau)}{(1-p(\tau))}>0
$$

So

$$
\frac{d V}{d t} \leq-\beta V+\alpha
$$

This leads us to:

$$
\begin{gathered}
-\frac{1}{\beta} \int_{t_{0}}^{t_{r}} \frac{d V}{V-\frac{\alpha}{\beta}} \leq \int_{t_{0}}^{t_{r}} d t \\
-\frac{1}{\beta}\left[\ln \left|V\left(t_{r}\right)-\frac{\alpha}{\beta}\right|-\ln \left|V\left(t_{0}\right)-\frac{\alpha}{\beta}\right|\right] \leq\left(t_{r}-t_{0}\right)
\end{gathered}
$$

Therefore

$$
t_{r}=t_{0}+\frac{\ln \left|V\left(t_{0}\right)-\frac{\alpha}{\beta}\right|-\ln \left|V\left(t_{r}\right)-\frac{\alpha}{\beta}\right|}{\beta}
$$

By assumption: Since, the condition in Theorem 2 is satisfied. thus in terms of theorem 2, There is a global synchronization at a finite time between the three oscillators $X, Y$ and $Z$.

\section{Numerical Simulations}

The aim here is to investigate the synchronization behavior that can be observed in the relay coupling of three NHOtype oscillators. To achieve this, we numerically integrated systems (3), (4) and (5) by using the standard fourth-order RungeCKutta method with time step equal to $\Delta t=0.01$. It is worth to recall that we select the parameters of NHO as $k_{1}=0.9, k_{2}=0.93, k_{3}=0.82, k_{4}=0.93$, $k_{5}=0.84, k_{6}=0.93, e=25$ and so that the system (2) exhibits bursting oscillations. Let us note that initial values of systems (3), (4) and (5) are respectively given as follows: $X(0)=(0.001,0.008,0.004,0.004,0.005,0.01)^{T}$, $Y(0)=(0.51,0.38,0.24,0.14,0.25,0.61)^{T}$ and $Z(0)=$ $(1.001,1.08,1.04,1.004,1.05,1.01)^{T}$.

\subsection{Non-adaptive Case}

The numerical results simulations for the case of nonadaptive synchronization are presented in this part. Figure 4 depicts the dynamics of synchronization error $(e=X-Z)$ between the outer oscillators $X$ and $Z$ (Figure 4(a)) with the 
control gain $\bar{d}=5$. In Figure 4(a), we find that between the two outer oscillators $X$ and $Z$, the synchronization error is almost zero from $t=200$. Which shows that through the relay unit $Y$, there is a complete synchronization (CS) between the oscillators $X$ and $Z$. This result is confirmed in Figure 4(b) which is time series of trajectories $X$ in blue, and $Z$ in black. Meanwhile, other numerical results are presented in Figure 5. We see in Figure 5(a), the time series of the synchronization errors $(e=X+Z-2 Y)$ and $(e=X-Z)$ respectively between the three oscillators $X, Y$ and $Z$ given in blue and on the other hand between the two outer oscillators $X$ and $Z$ given in black. Almost from $t=200$, we notice on this curve that the errors are zero, which shows that there is indeed global synchronization (GS) in the system. This result is confirmed in Figure 5(b), which is the time representation of states of oscillators $X, Y$ and $Z$ with $X$ in blue, $Y$ in red, $Z$ in black. Furthermore, the bursting oscillations are always observed in the relay configuration of the three oscillators (see Figure 6). Figure 6(a) presents Time series of the variable $y_{1}(t)$ whereas Figure 6(b) shows the phase portrait in the plane $\left(y_{1}-y_{2}\right)$.

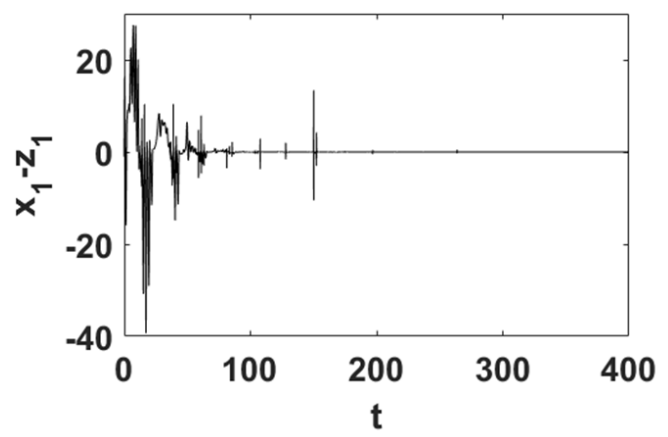

(a)

\subsection{Adaptive Case}

Here, Figure 7 shows time series synchronization error between the outer oscillators $X$ and $Z$ (see Figure 7(a)). With regard to the latter (i.e, Figure 7(a)), we see that this synchronization error is canceled from $t=50$ : we speak in this case of complete synchronization (CS). Figure 7(b) (which is time series of state variables $X$ in blue and $Z$ in black) confirms these results, for the parameter value $\bar{d}=$ 0.001. Similarly, Figure 8 displays the dynamics of the synchronization errors (see Figure 8(a)) and (the time series of state variables $X, Y$ and $Z$ (see Figure 8(b)) always for $\bar{d}=0.001$. we notice in Figure 8 (a) that the synchronization errors between the outer oscillators $X$ and $Z$ in black and between all three coupled oscillators $X, Y$ and $Z$ in blue converge very quickly to zero: hence the robustness of the adaptive controller. This result is validated by the Figure $8(\mathrm{~b})$ which is the time representation of states of oscillators $X$ in blue, $Y$ in red and $Z$ in black. Figure 9 shows changing the adaptive control parameter $d_{i}$. The initial value of $d_{i}$ is zero and $\bar{d}=0.001$.

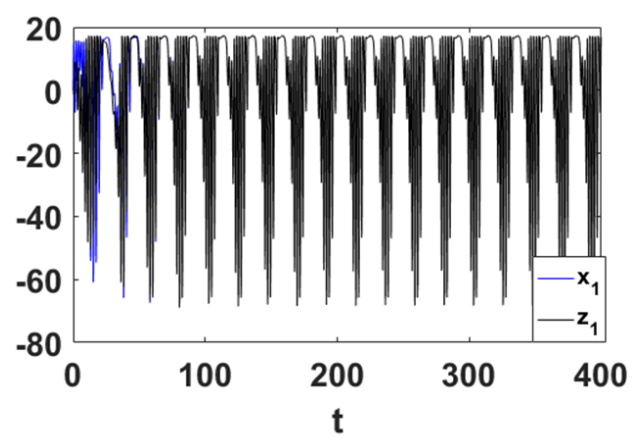

(b)

Figure 4. Complete synchronization (CS) in a relay coupling of three NHO-type oscillators. (a) Time series of the trajectory of non-adaptive synchronization error between the outer oscillators 1 and $2\left(e=x_{1}-z_{1}\right)$. (b) Time series of trajectories $x_{1}$ in blue, $z_{1}$ in black for the parameter value $\bar{d}=5$.

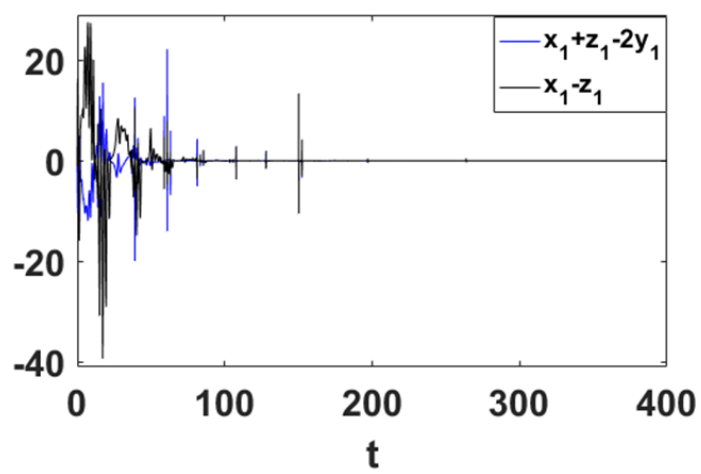

(a)

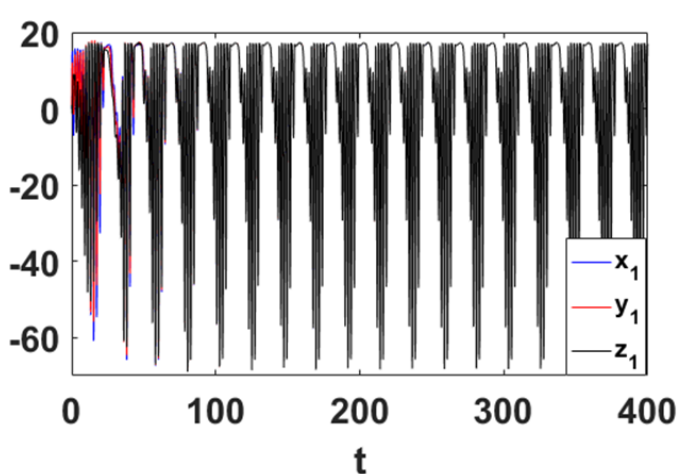

(b)

Figure 5. Global synchronization (GS) in a relay coupling of three NHO-type oscillators. (a) Time series of trajectories of non-adaptive synchronization errors $e=x_{1}+z_{1}-2 y_{1}$ in blue and $e=x_{1}-z_{1}$ in black. (b) Time series of trajectories $x_{1}$ in blue, $y_{1}$ in red, $z_{1}$ in black for the parameter value $\bar{d}=5$. 


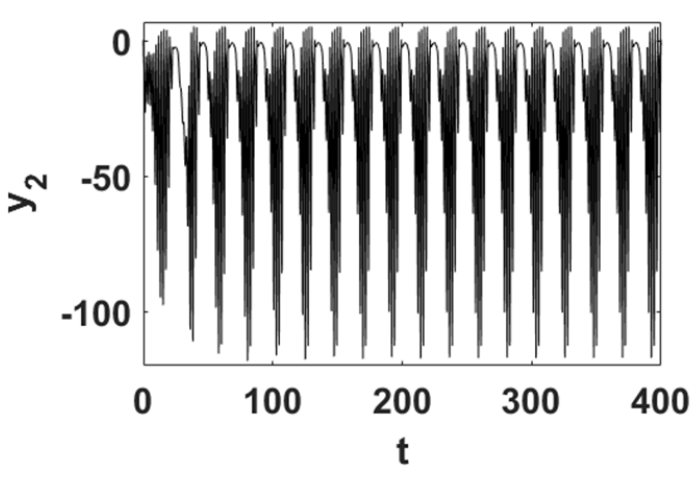

(a)

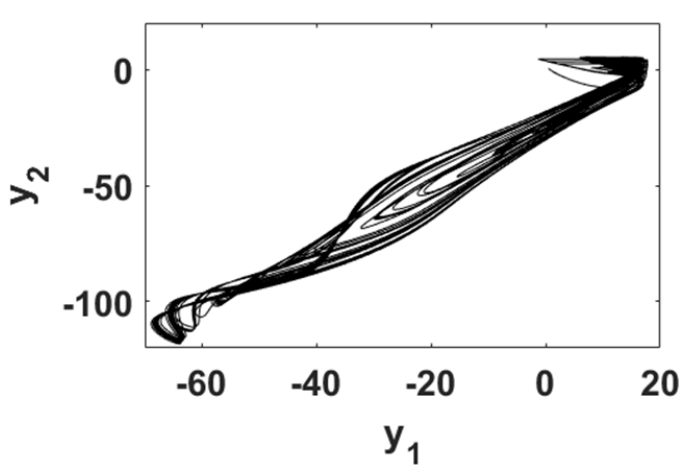

(b)

Figure 6. Numerical simulation of bursting oscillations in a relay coupling of three NHO-type oscillators, for the parameter value $k=5:$ (a) Time series of the variable $y_{1}(t)$. (b) Phase portrait in the plane $\left(y_{1}-y_{2}\right)$.

\section{Circuit Realization in a Relay Coupling of Three NHO-type Oscillators}

To confirm the implementation of our strategy, we performed simulations in the PSpice software. Above all, it is important for us to remember that, PSpice (Personal simulation program with integrated circuit emphasis) analysis is one of the most common approaches used in nonlinear electronics to validate theoretical results [32]. Indeed, PSpice offers a suitable environment where electronic circuit is built up and further simulated as in real time experiment [40]. One of the main advantage of such approach is that one can easily choose the suitable electronics components which will further being used either in numerical analysis or in real experimental investigations $[32,41]$. In the rest of the work, we consider the following values for the key parameters which have been used for numerical and PSpice results in [32]: $C_{G S}=3.736 p F, C_{G D}=3.35 p F, I_{S}=34.57 p A$, $V_{G S o f f}=-1.409 \mathrm{~V}, V_{T}=25 \mathrm{mV}$, and $g_{m 0}=1.754 \mathrm{mAV}^{-2}$, $I_{0}=1 \mathrm{~mA}, \omega_{0}=10^{7} \mathrm{rad} / \mathrm{s}, C=20 \mathrm{nF}, L_{1}=24.5 \mathrm{uH}$, $L_{2}=4 u H, L_{3}=0.7 u H, L_{4}=0.2 u H, E=0.6255 \mathrm{~V}$ and considering the mutual coupling between inductances as $k_{1}=0.9, k_{2}=0.93, k_{3}=0.82, k_{4}=0.93, k_{5}=$ 0.84 and $k_{6}=0.93$. For this synchronization approach, we will only be interested in its non adaptive method. The circuit of Figure 10 realizes the non-adaptive synchronization of the relay coupling of three Identical NHOs in PSpice, with for initial conditions: $(0,0,0.002,0.1,0.001,0.0001)$ for the outer 1 oscillator, $(0,0,0.002,0.01,0.001,0.01)$ for the outer 2 oscillator and $(0,0,0.2,0.01,0.1,0.1)$ for the relay unit.

From the description above, the dynamical behavior of the system Figure 10 is mathematically described by:

Outer 1 oscillator:

$$
\left\{\begin{aligned}
\frac{d x_{1}}{d t} & =\frac{I_{0}}{V_{T} C_{G S} \omega_{0}}\left(x_{5}-x_{4}\right)-\frac{I_{s}}{V_{T} C_{G S} \omega_{0}}\left[\exp \left(x_{1}\right)-1\right]-\frac{g_{m 0} V_{T}}{C_{G S} \omega_{0}} g\left(x_{1}, x_{2}\right)+\frac{1}{C_{G S} \omega_{0} R_{a}}\left(y_{1}-x_{1}\right) \\
\frac{d x_{3}}{d t} & =-\frac{I_{0} C_{G D} \omega_{0}}{V_{5} C \omega_{0}} x_{6}+\frac{\alpha g_{m 0} V_{T}}{C_{G D} \omega_{0}} g\left(x_{1}, x_{2}\right) \\
\frac{d x_{4}}{d t} & =b_{1} x_{1}+b_{2}\left(\frac{E}{V_{T}}+x_{2}\right)+b_{3} x_{3} \\
\frac{d x_{5}}{d t} & =b_{4} x_{1}+b_{5}\left(\frac{E}{V_{T}}+x_{2}\right)+b_{6} x_{3} \\
\frac{d x_{6}}{d t} & =b_{7} x_{1}+b_{8}\left(\frac{E}{V_{T}}+x_{2}\right)+b_{9} x_{3}
\end{aligned}\right.
$$

Relay oscillator:

$$
\left\{\begin{aligned}
\frac{d y_{1}}{d t} & =\frac{I_{0}}{V_{T} C_{G S} \omega_{0}}\left(y_{5}-y_{4}\right)-\frac{I_{s}}{V_{T} C_{G S} \omega_{0}}\left[\exp \left(y_{1}\right)-1\right]-\frac{g_{m 0} V_{T}}{C_{G S} \omega_{0}} g\left(y_{1}, y_{2}\right)+\frac{1}{C_{G S} \omega_{0} R_{a} R_{b}}\left[R_{b} x_{1}+R_{a} z_{1}-\left(R_{a}+R_{b}\right) y_{1}\right] \\
\frac{d y_{3}}{d t} & =\frac{I_{0} C_{G D} \omega_{0}}{V_{T} C \omega_{0}} y_{6}+\frac{\alpha g_{m 0} V_{T}}{C_{G D} \omega_{0}} g\left(y_{1}, y_{2}\right) \\
\frac{d y_{4}}{d t} & =b_{1} y_{1}+b_{2}\left(\frac{E}{V_{T}}+y_{2}\right)+b_{3} y_{3} \\
\frac{d y_{5}}{d t} & =b_{4} y_{1}+b_{5}\left(\frac{E}{V_{T}}+y_{2}\right)+b_{6} y_{3} \\
\frac{d y_{6}}{d t} & =b_{7} y_{1}+b_{8}\left(\frac{E}{V_{T}}+y_{2}\right)+b_{9} y_{3}
\end{aligned}\right.
$$


Outer 2 oscillator:

$$
\left\{\begin{aligned}
\frac{d z_{1}}{d t} & =\frac{I_{0}}{V_{T} C_{G S} \omega_{0}}\left(z_{5}-z_{4}\right)-\frac{I_{s}}{V_{T} C_{G S} \omega_{0}}\left[\exp \left(z_{1}\right)-1\right]-\frac{g_{m 0} V_{T}}{C_{G S} \omega_{0}} g\left(z_{1}, z_{2}\right)+\frac{1}{C_{G S} \omega_{0} R_{b}}\left(y_{1}-z_{1}\right) \\
\frac{d z_{3}}{d t} & =-\frac{I_{0}}{V_{0} C_{G D} \omega_{0}} z_{5}+\frac{\alpha g_{m 0} V_{T}}{C_{G} \omega_{0}} g\left(z_{1}, z_{2}\right) \\
\frac{d z_{4}}{d t} & =b_{1} z_{1}+b_{2}\left(\frac{E}{V_{T}}+z_{2}\right)+b_{3} z_{3} \\
\frac{d z_{5}}{d t} & =b_{4} z_{1}+b_{5}\left(\frac{E}{V_{T}}+z_{2}\right)+b_{6} z_{3} \\
\frac{d z_{6}}{d t} & =b_{7} z_{1}+b_{8}\left(\frac{E}{V_{T}}+z_{2}\right)+b_{9} z_{3}
\end{aligned}\right.
$$

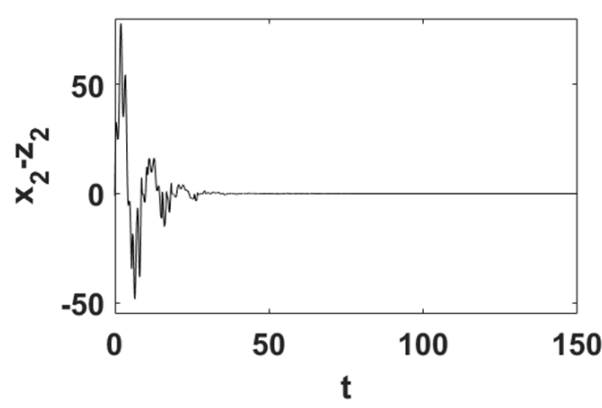

(a)

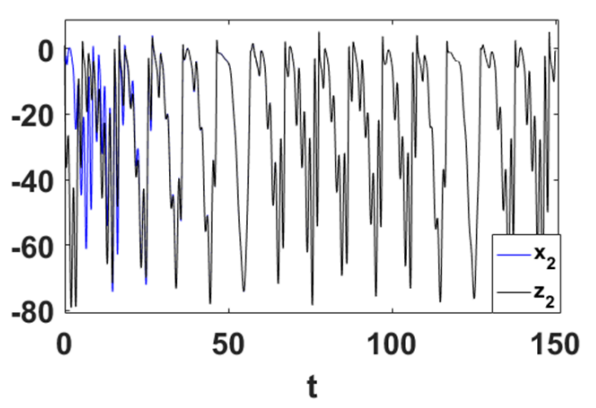

(b)

Figure 7. Complete synchronization (CS) in a relay coupling of three NHO-type oscillators. (a) Time series of the trajectory of adaptive synchronization error between the outer oscillators 1 and $2\left(e=x_{1}-z_{1}\right)$. (b) Time series of trajectories $x_{2}$ in blue, $z_{2}$ in black for the parameter value $\bar{d}=0.001$.

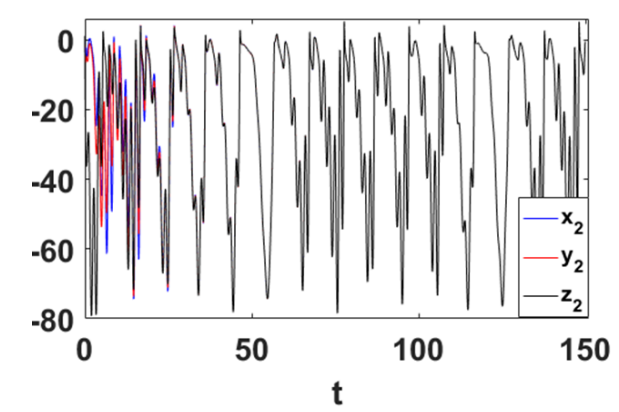

(a)

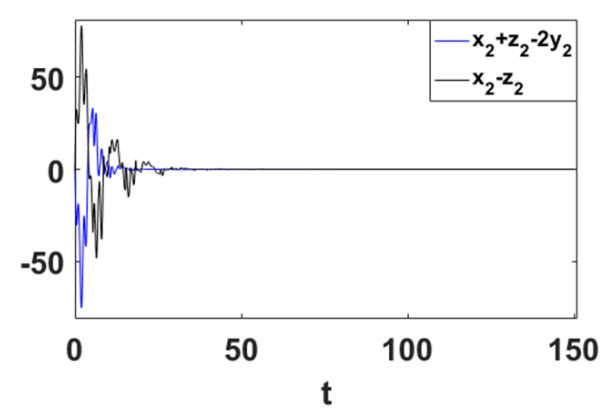

(b)

Figure 8. Global synchronization (GS) in a relay coupling of three NHO-type oscillators. (a) Time series of trajectories of adaptive synchronization errors $e=x_{2}+z_{2}-2 y_{2}$ in blue and $e=x_{2}-z_{2}$ in black. (b) Time series of trajectories $x_{2}$ in blue, $y_{2}$ in red, $z_{2}$ in black for the parameter value $\bar{d}=0.001$.

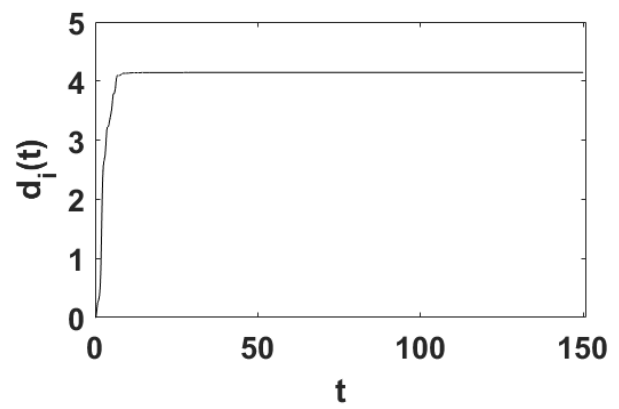

Figure 9. Changing of adaptive control parameter $d_{i}$ with time $t$. 
In this circuit, the resistors $R_{a}$ and $R_{b}$ are the coupling resistances. By identification between systems (3), (4) and (5) and systems (58), (59) and (60), The relationship between $\bar{d}$ (coupling parameter) and resistors $R_{a}$ and $R_{b}$ are:

$$
R_{a}=R_{b}=\frac{1}{\bar{d} C_{G S} \omega_{0}}
$$

Using the numerical simulation values, the circuit parameters $R_{a}$ and $R_{b}$ are obtained: $R_{a}=R_{b}=5.353 \mathrm{k} \Omega$

Thus, for parameters used to find global synchronization results found in Figure 4, the circuit is simulated with PSpice software and the obtained results are depicted in Figure 11. We note that, these latters are in full agreement with the numerical simulation obtained in MATLAB software.

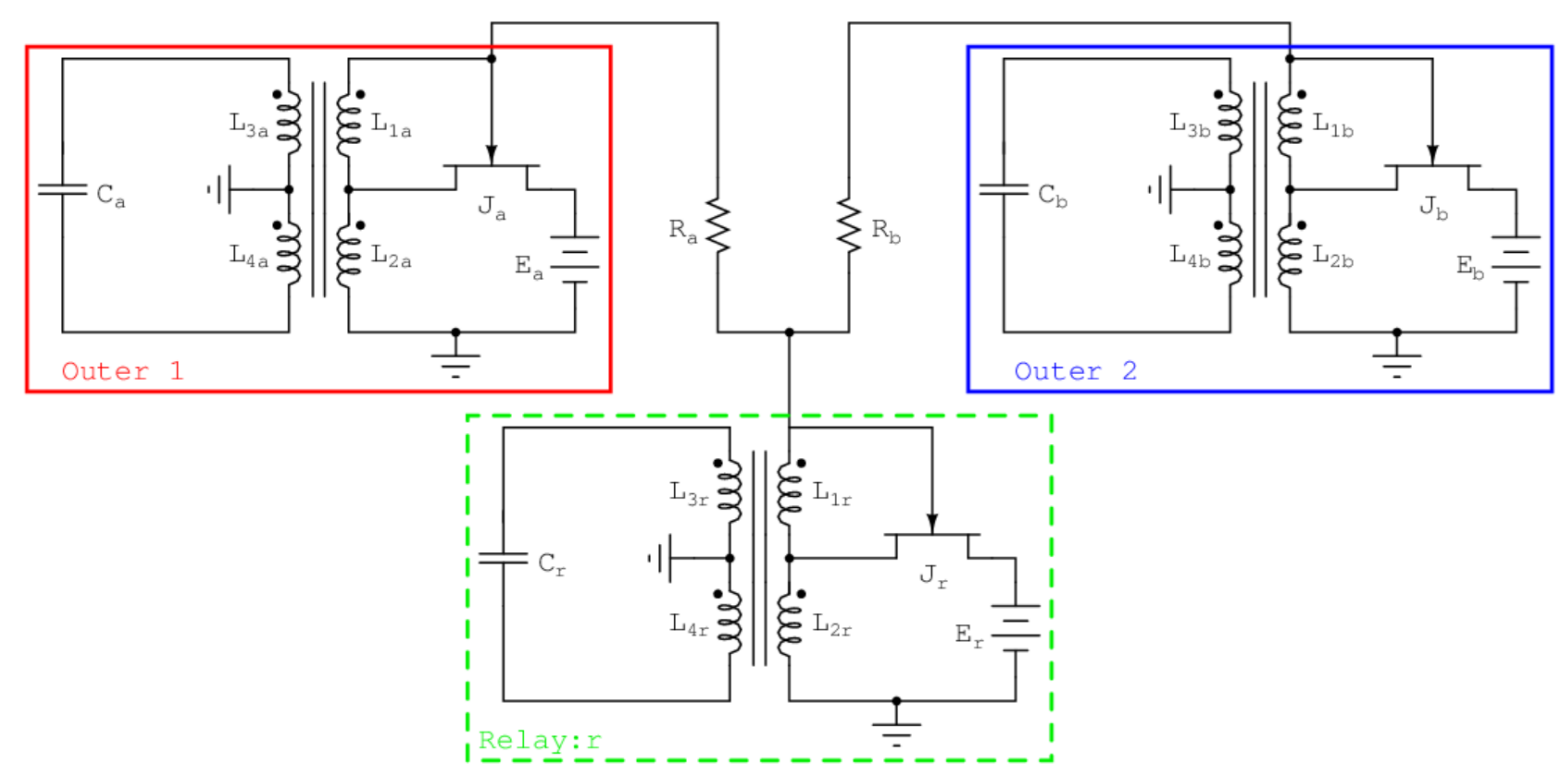

Figure 10. Circuit performing the non-adaptive synchronization in a relay coupling of three NHO-type oscillators.

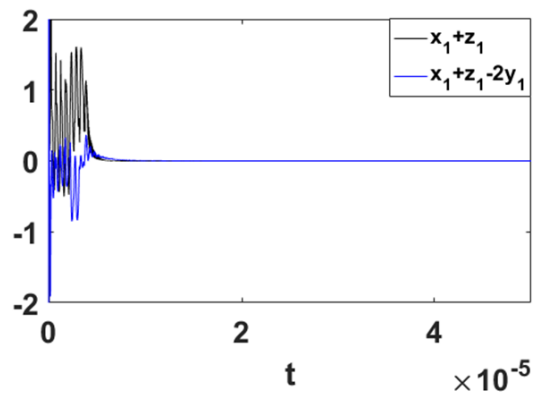

(a)

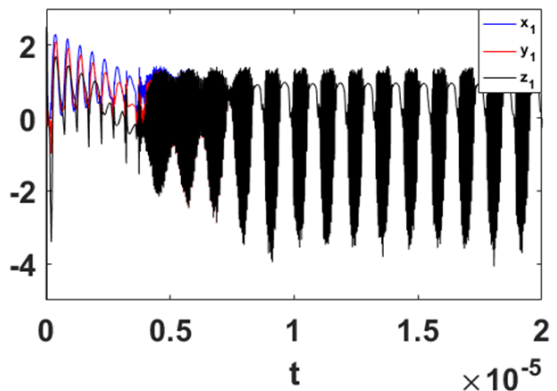

(b)

Figure 11. Global synchronization (GS) in PSpice. (a) Time series of trajectories: $e=x_{1}-z_{1}$ in blue and $e=x_{1}+z_{1}-2 y_{1}$ in red. (b) Time series of trajectories $x_{1}$ in blue, $y_{1}$ in red, $z_{1}$ in black for the values $R_{a}=R_{b}=100 \Omega$.

\section{Conclusion}

The NHO-type oscillator operating at high frequency introduced in [32] has been recalled in this study. Among the complex behaviours exhibited by this oscillator, we have limited our investigation to the set of parameters for which our oscillator presented a rich dynamic phenomenon intrinsic to neurons, characterized by alternating sequence of active states and passive states, and known as bursting oscillations. Furthermore, we have demonstrated that it is possible to determine a relay coupling scheme of three NHOtype oscillators. From this configuration, the synchronization issue has been studied. The control technique proposed in this paper known as PIDOC [25] results from the combination between the periodically intermittent control and discrete-time state observations. Applying the Lyapunov method to the two synchronization cases (non-adaptive and adaptive) discussed above, sufficient conditions of stability have been obtained 
for a better guarantee of the synchronization of three relaycoupled NHO-type oscillators.

More importantly, the synchronization time for the adaptive case has been foretell by calculation because this time has to be known and minimized, so as to achieve the synchronization as fast as possible. From the numerical results, we obtained a global synchronisation between all three NHOtype relay coupled oscillators from both the non-adaptive synchronization and the adaptive synchronization methods, thus validating conditions of analytical stability for global synchronization. For non-adaptive case, this phenomenon of global synchronisation was further confirmed using electronic implementation in the PSpice environment. PSpice analysis reveals good correspondence with numerical investigations. Thus, in further works on this topic, we will employ PIDOC to investigate synchronization of fractional-order of three relay coupled NHO-type oscillators. Furthermore, the relay configuration which was examined in this paper can also be used in big data technologies to encrypt and compress data [42, 43, 44].

\section{References}

[1] Pikovsky A., Rosenblum M., Kurths J. (2002) Synchronization: a universal concept in nonlinear science.

[2] Sharma A, Shrimali M. D., Aihara K. (2014) Effect of mixed coupling on relay-coupled Rössler and lorenz oscillators, Physical Review E 90 (6), 062907.

[3] koseska A., Volkov E., and Kurths J. (2013) Oscillation quenching mechanisms: Amplitude vs. oscillation death, Physics Reports 531 (4), 173-199.

[4] Saxena G., Prasad A., Ramaswamy R. (2012) Amplitude death: The emergence of stationarity in coupled nonlinear systems, Physics Reports 521 (5), $205 \mathrm{C} 228$.

[5] Karnatak R, Punetha N, Prasad A, Ramaswamy R (2010) Nature of the phase-flip transition in the synchronized approach to amplitude death, Physical Review E 82 (4), 046219.

[6] Kerimoğlu D. Synchronization of pendulum like systems, Ph.D. thesis, bilkent university (2011).

[7] Pecora L. M., Carroll T. L. (1990) Synchronization in chaotic systems, Physical review letters 64 (8), 821.

[8] Tamba V. K., Fotsin H., Kengne J., Tagne F. K., Talla P. (2015) Coupled inductors-based chaotic colpitts oscillators: Mathematical modeling and synchronization issues, The European Physical Journal Plus 130 (7), 137.

[9] Li S., Xu W., Li R. (2007) Synchronization of two different chaotic systems with unknown parameters, Physics Letters A 361 (1-2), 98C102.
[10] Fotsin H., Daafouz J. (2005) Adaptive synchronization of uncertain chaotic colpitts oscillators based on parameter identification, Physics Letters A 339 (3-5), 304C315.

[11] Thounaojam U. S., Shrimali M. D. (2018) Phase-flip in relay oscillators via linear augmentation, Chaos, Solitons \& Fractals 107, 5C12.

[12] Liu D., Ye D. (2018) Anti-synchronization of neural networks with mixed delays, in: International Conference on Neural Information Processing, Springer, pp. $361 \mathrm{C} 370$.

[13] Naderi B., Kheiri H., Heydari A. (2018) Antisynchronization of complex chaotic T-system via optimal adaptive sliding-mode and its application in secure communication, International Journal of Industrial Mathematics 10 (2), 181C192.

[14] Cai G., Zhang Z., Feng G., Chen Q. (2019) Delay feedback impulsive control of a time-delay nonlinear complex financial networks, Indian Journal of Physics, 1 C6.

[15] Karnatak R., Ramaswamy R., Prasad A. (2007) Amplitude death in the absence of time delays in identical coupled oscillators, Physical Review E 76 (3), 035201.

[16] Karnatak R., Ramaswamy R., Prasad A. (2009) Synchronization regimes in conjugate coupled chaotic oscillators, Chaos: An Interdisciplinary Journal of Nonlinear Science 19 (3), 033143.

[17] Sharma A., Shrimali M. D., Prasad A., Ramaswamy R., Feudel U. (2011) Phase-flip transition in relay-coupled nonlinear oscillators, Physical Review E 84 (1), 016226.

[18] Banerjee R., Ghosh D., Padmanaban E., Ramaswamy R., Pecora L., Dana S. K. (2012) Enhancing synchrony in chaotic oscillators by dynamic relaying, Physical Review E 85 (2), 027201.

[19] Gutiérrez R., Sevilla-Escoboza R., Piedrahita P., Finke C., Feudel U., Buldu J. M., Huerta-Cuellar G., JaimesReategui R., Moreno Y., Boccaletti S. (2013) Generalized synchronization in relay systems with instantaneous coupling, Physical Review E 88 (5), 052908.

[20] Zhao N., Sun Z., Xu W. (2018) Amplitude death induced by mixed attractive and repulsive coupling in the relay system, The European Physical Journal B 91 (1), 20.

[21] Louodop P., Ngouonkadi E. B. M., Muruganandam P., Cerdeira H. A. (2018) Synchronization dynamics of modified relay-coupled chaotic systems, Journal of Applied Nonlinear Dynamics 7 (1), 11C24.

[22] Kengne R., R. Tchitnga, S. Mabekou, B. R. W. Tekam, G. B. Soh, A. Fomethe, On the relay coupling of three fractional-order oscillators with time-delay consideration: Global and cluster synchronizations, Chaos, Solitons \& Fractals 111 (2018), 6C17. 
[23] Sharma A. (2019) Time delay induced partial death patterns with conjugate coupling in relay oscillators, Physics Letters A 383 (16), 1865C1870.

[24] Zhang L., Yang Y. (2017) Lag synchronization for fractional-order memristive neural networks via period intermittent control, Nonlinear Dynamics 89 (1), $367 \mathrm{C} 381$.

[25] Xu Y., Li Q., Li W. (2019) Periodically intermittent discrete observation control for synchronization of fractional-order coupled systems, Communications in Nonlinear Science and Numerical Simulation 74, $219 \mathrm{C} 235$.

[26] Fan Y., Huang X., Wang Z., Xia J., Li Y. (2018) Global mittag-leffler synchronization of delayed fractionalorder memristive neural networks, Advances in Difference Equations 2018 (1), 338.

[27] Matouk A. (2011) Chaos, feedback control and synchronization of a fractional-order modified autonomous van der polCduffing circuit, Communications in Nonlinear Science and Numerical Simulation 16 (2), 975C986.

[28] Wan P., Jian J, Mei J. (2018) Periodically intermittent control strategies for $\alpha$-exponential stabilization of fractional-order complex-valued delayed neural networks, Nonlinear Dynamics 92 (2), 247C265.

[29] Li H. L., Hu C., Jiang H., Teng Z., Jiang Y. L. (2017) Synchronization of fractional-order complex dynamical networks via periodically intermittent pinning control, Chaos, Solitons \& Fractals 103, 357C363.

[30] Nguimdo R. M., Tchitnga R., Woafo P. (2013) Dynamics of coupled simplest chaotic two-component electronic circuits and its potential application to random bit generation, Chaos: An Interdisciplinary Journal of Nonlinear Science 23 (4), 043122.

[31] Tekam R. B. W., Kengne J., Kenmoe G. D. (2019) High frequency Colpitts' oscillator: A simple configuration for chaos generation, Chaos, Solitons \& Fractals 126, $351 \mathrm{C} 360$.

[32] Tchitnga R., Mezatio B., Fozin T. F., Kengne R., Fotso P. L., Fomethe A. (2019) A novel hyperchaotic three-component oscillator operating at high frequency, Chaos, Solitons \& Fractals 118, 166C180. https://doi.org/10.1016/j.chaos.2018.11.015

[33] Kengne R., Tchitnga R, Mezatio A., Fomethe A, Litak G. (2017) Finite-time synchronization of fractionalorder simplest two-component chaotic oscillators, The European Physical Journal B 90 (5), 88.

[34] Louodop P., Fotsin H., Kountchou M., Bowong S. (2013) Finite-time synchronization of lorenz chaotic systems: theory and circuits, Physica Scripta 88 (4), 045002.
[35] Louodop P., Kountchou M., Fotsin H., Bowong S. (2014) Practical finite-time synchronization of jerk systems: theory and experiment, Nonlinear Dynamics 78 (1), $597 \mathrm{C} 607$.

[36] Kengne R., Tchitnga R., Fomethe A., Hammouch Z. (2017) Generalized finite-time function projective synchronization of two fractional-order chaotic systems via a modified fractional nonsingular sliding mode surface, Commun Numer Anal, 233C248.

[37] Kengne R., Tchitnga R., Kouanou A. T., Fomethe A. (2013) Dynamical properties and chaos synchronization in a fractional-order two-stage colpitts oscillator, Journal of Engineering Science \& Technology Review 6 (4).

[38] Wu R., Lu Y., Chen L. (2015) Finite-time stability of fractional delayed neural networks, Neurocomputing 149 , $700 \mathrm{C} 707$.

[39] Kuczma M. (2009) An introduction to the theory of functional equations and inequalities: Cauchy's equation and Jensens inequality, Springer Science \& Business Media.

[40] Mezatio B. A., Motchongom M. T., Tekam B. R. W., Kengne R., Tchitnga R., Fomethe A. (2019) A novel memristive 6d hyperchaotic autonomous system with hidden extreme multistability, Chaos, Solitons \& Fractals 120, 100C115. https://doi.org/10.1016/j.chaos.2019.01.015

[41] Mezatio B. A., Tingue M. M., Kengne R., Kouanou A. T., Fonzin T. F., Tchitnga R. (2019) Complex dynamics from a novel memristive $6 \mathrm{~d}$ hyperchaotic autonomous system, International Journal of Dynamics and Control, 1C21. https://doi.org/10.1007/s40435-019-00531-y

[42] Usama M., Zakaria N. (2017) Chaos-based simultaneous compression and encryption for hadoop, PloS one 12 (1), e0168207.

[43] Kouanou A. T., Tchiotsop D., Kengne R., Zephirin D. T., Armele N. M. A., Tchinda R. (2018) An optimal big data workflow for biomedical image analysis, Informatics in Medicine Unlocked 11, 68C74

[44] Kouanou A. T., Tchiotsop D., Tchinda R., Tchapga C. T., Telem A. N. K., Kengne R. (2018) A machine learning algorithm for biomedical images compression using orthogonal transforms, International Journal of Image, Graphics \& Signal Processing 10 (11).

[45] Kouanou A. T., Tchiotsop D., Fozin T. F., Mounmo B., Tchinda R. (2019) Real-time image compression system using an embedded board, Science Journal of Circuits, Systems and Signal Processing 7 (4), 81. DOI: 10.11648/j.cssp.20180704.11 